\title{
Coordinated Clearance of Periciliary Liquid and Mucus from Airway Surfaces
}

\author{
Hirotoshi Matsui, ${ }^{\star}$ Scott H. Randell, ${ }^{\star}$ Steven W. Peretti, ${ }^{\S}$ C. William Davis, ${ }^{\star \ddagger}$ and Richard C. Boucher* \\ *Cystic Fibrosis/Pulmonary Research and Treatment Center, and ${ }^{\ddagger}$ Departments of Medicine and Physiology, University of North Carolina \\ at Chapel Hill, Chapel Hill, North Carolina 27599; and ${ }^{\S}$ Department of Chemical Engineering, North Carolina State University, Raleigh, \\ North Carolina 27695
}

\begin{abstract}
Airway surface liquid is comprised of mucus and an underlying, watery periciliary liquid (PCL). In contrast to the well-described axial transport of mucus along airway surfaces via ciliary action, theoretical analyses predict that the PCL is nearly stationary. Conventional and confocal microscopy of fluorescent microspheres and photoactivated fluorescent dyes were used with well-differentiated human tracheobronchial epithelial cell cultures exhibiting spontaneous, radial mucociliary transport to study the movements of mucus and PCL. These studies showed that the entire PCL is transported at approximately the same rate as mucus, 39.2 \pm 4.7 and 39.8 $\pm 4.2 \mu \mathrm{m} / \mathrm{sec}$, respectively. Removing the mucus layer reduced PCL transport by $>80 \%$, to $4.8 \pm 0.6 \mu \mathrm{m} / \mathrm{sec}$, a value close to that predicted from theoretical analyses of the ciliary beat cycle. Hence, the rapid movement of PCL is dependent upon the transport of mucus. Mucus-dependent PCL transport was spatially uniform and exceeded the rate expected for pure frictional coupling with the overlying mucus layer; hence, ciliary mixing most likely accelerates the diffusion of momentum from mucus into the PCL. The cephalad movement of PCL along airway epithelial surfaces makes this mucus-driven transport an important component of salt and water physiology in the lung in health and disease. (J. Clin. Invest. 1998. 102:11251131.) Key words: lung • airway • cilia $\bullet$ mucus $\bullet$ mucin $\bullet$ mucociliary clearance $\bullet$ airway surface liquid
\end{abstract}

\section{Introduction}

The airway surface liquid (ASL) ${ }^{1}$ that covers the epithelia lining mammalian airways constitutes a first line of defense against inhaled pathogens. It appears to be comprised of two relatively distinct layers (1): a watery layer that surrounds the cilia on the apical surface of the ciliated cell, i.e., the periciliary liquid layer (PCL); and a layer rich in mucins, the mucus layer, that resides atop the PCL. Despite advances in our understanding of the

Address correspondence to Dr. C. William Davis, 6009 ThurstonBowles Building, CB\#7248, University of North Carolina at Chapel Hill, Chapel Hill, NC 27599. Phone: 919-966-7060; FAX: 919-9667524; E-mail: cwdavis@med.unc.edu

Received for publication 30 December 1997 and accepted in revised form 16 July 1998.

1. Abbreviations used in this paper: ASL, airway surface liquid; hTBE, human tracheobronchial epithelial; PCL, periciliary liquid layer.

J. Clin. Invest.

(C) The American Society for Clinical Investigation, Inc. 0021-9738/98/09/1125/07 \$2.00

Volume 102, Number 6, September 1998, 1125-1131

http://www.jci.org physical topography of ASL (2-5), the physiologic processes that regulate the properties of ASL are not understood.

Extensive studies have suggested that airway epithelia regulate the composition and/or volume of ASL $(6,7)$. There is controversy, however, over the relative importance of compositional versus volume regulation. One view $(8,9)$, the hypotonic-defensin hypothesis, holds that airway epithelia normally regulate the composition, i.e., tonicity, of ASL, whereas another view, the isotonic volume absorbing hypothesis (10), suggests that isotonic volume regulation of ASL is the primary function of airway epithelia. These two views have very different predictions of the macroscopic physiology on airway surfaces. The hypotonic-defensin hypothesis, for instance, predicts that PCL remains stationary, whereas the mucus layer is transported, which is consistent with predictions based on ciliary stroke analyses (11-15). In contrast, the alternative hypothesis argues that the ASL isotonic volume regulatory function of airway epithelia reflects the cephalad movement of both PCL and mucus up the converging surface area of mammalian airways.

It has heretofore not been possible to separate and measure the transport of discrete PCL and mucus layers in mammalian airway epithelia, in part due to the very shallow depths of these layers. Recent advances in culture technique have resulted in the routine production of well-differentiated human tracheobronchial epithelial (hTBE) cultures that produce mucus and transport it radially over their luminal surfaces. We have used these cultures to label the periciliary and mucus layers of ASL by using fluorescent markers and to measure the real time transport of each layer by using vertical profile sections (xz) obtained by confocal microscopy.

\section{Methods}

Cell culture. Well-differentiated human tracheobronchial epithelial cultures were produced using Costar 12-mm Transwell-Col supports according to the general procedure of Gray et al. (16). Cells were removed by protease dissociation (17) from portions of main stem or lobar bronchi representing excess donor tissue obtained at the time of lung transplantation under the auspices of the UNC Institutional Committee on the Protection of The Rights of Human Subjects. Dissociated primary airway epithelial cells were grown on $100-\mathrm{mm}$ tissue culture dishes in modified BEGM media (18), which consisted of LHC Basal Media (Biofluids Inc., Rockville, MD) supplemented with insulin $(5 \mu \mathrm{g} / \mathrm{ml})$, hydrocortisone $(0.072 \mu \mathrm{g} / \mathrm{ml})$, EGF $(25 \mathrm{ng} / \mathrm{ml}), \mathrm{T}_{3}$ $\left(10^{-8} \mathrm{M}\right)$, transferrin $(10 \mu \mathrm{g} / \mathrm{ml})$, epinephrine $(0.6 \mu \mathrm{g} / \mathrm{ml})$, phosphoethanolamine $(0.5 \mu \mathrm{M})$, ethanolamine $(0.5 \mu \mathrm{M})$, bovine pituitary extract $\left(0.8 \%\right.$; see [19]), BSA $(0.5 \mathrm{mg} / \mathrm{ml}), \mathrm{CaCl}_{2}(80 \mu \mathrm{M})$, trace elements $(1 \times$; Biofluids Inc.), Stock $4(1 \times$; Biofluids Inc.), Stock $11(1 \times$; Biofluids Inc.), gentamicin $(50 \mu \mathrm{g} / \mathrm{ml})$, penicillin (50 units $/ \mathrm{ml})$, streptomycin $(50 \mathrm{ng} / \mathrm{ml})$, and retinoic acid $\left(5 \times 10^{-8} \mathrm{M}\right)$. Passage one cells were seeded on the T-Col membranes at a density of $0.25 \times 10^{6} / \mathrm{cm}^{2}$, and modified BEGM media was used in which the base mixture was 50:50 LHC Basal and DMEMH, instead of 100\% LHC Basal Media; the EGF concentration was reduced to $0.5 \mathrm{ng} / \mathrm{ml}$, and gentamicin and amphotericin B were omitted. Upon confluence, the apical surface 
was rinsed with PBS and medium was only replaced in the basolateral chamber. Cultures were used when they were fully differentiated and producing mucus, 2-4 wk following confluence. Preparations were screened for rotational mucus transport by using fluorescence microscopy to examine the behavior of $1-\mu \mathrm{m}$ fluorescent beads $(0.02 \%$ in 50 $\mu \mathrm{l}$ of PBS; see below) on culture surfaces. Approximately $25 \%$ of cultures exhibited rotational mucus transport and were selected for study.

Labeling of ASL. The cells were stained vitally by a 15 -min exposure to $10 \mu \mathrm{M}$ calcein/AM (Molecular Probes, Eugene, OR). The surface liquid of living cultures was labeled with fluorescent latex microspheres $(0.02 \mathrm{vol} \%)$ and/or with dextrans $(10,000 \mathrm{D})$ conjugated to Texas Red or to DMNB caged-fluorescein (Molecular Probes) in 20 $\mu 1$ of PBS. Preliminary experiments revealed that addition of dithiothreitol (DTT; $10 \mathrm{mM})$ but not gelsolin $(0.5 \mu \mathrm{M})$, DNase $(0.25 \mathrm{U} / \mu \mathrm{l})$, or cytochalasin B $(1.0 \mu \mathrm{M})$ caused redistribution of beads into the PCL compartment and greatly reduced mucus transport rates $(20$, 21). We therefore developed a method to remove mucus from the culture surface by using $10 \mathrm{mM}$ DTT for $5 \mathrm{~min}$ followed by washing with 10 volumes of PBS. The effectiveness of this maneuver was verified by analysis of the mucus content of the serial washes by an ELISA technique (16). In experiments not shown, this DTT treatment had no significant effects on ciliary beat frequency (determined as described previously [22]).

Measurement of ASL transport. Cultures were observed by conventional, video epifluorescence microscopy (DMIRB microscope; C5985, cooled CCD camera; Leica; Hamamatsu), or by scanning con- focal microscopy (TCS 4D; Leica). The rate of rotational mucus transport was measured from 5-s images taken with the conventional fluorescence microscope of $1-\mu \mathrm{m}$ microspheres (Fig. $2 a$ ). The linear velocity was taken as the path lengths of individual beads measured along a radial originating at the apparent center of rotation (Fig. $2 b$ ). To measure bulk movement of ASL in these areas of rotational transport, caged-fluorescein dextrans were photoactivated with a 5-s exposure from a xenon or mercury lamp, through a DAPI filter, using a $40 \times$ objective. The rate of movement of the resulting circular areas of released fluorescein dextran fluorescence was measured in sequential images captured at intervals of 15-40 s (Fig. 3). By conventional fluorescence microscopy, the areas of released fluorescence were observed with a $5 \times$ objective, and in each pair of images obtained, the fluorescent area was traced, its centroid calculated, and its travel estimated as the linear distance between the two centroids. In contrast, by confocal microscopy, the released fluorescence was observed at trailing edge of the photoactivated spot with the same $40 \times$ objective used for photoactivation, as described in Results.

Dextran diffusion coefficients were determined by following the fluorescence decay after photoactivation using conventional fluorescence microscopy (23). Dextran diffusivity in PBS was determined using a solution placed between coverslips, whereas in mucus, it was determined in the surface liquid of a culture that possessed mucus but did not exhibit coordinated mucociliary clearance. The mucus layer in this culture, at $25 \mu \mathrm{m}$, was approximately fourfold thicker than the underlying PCL.
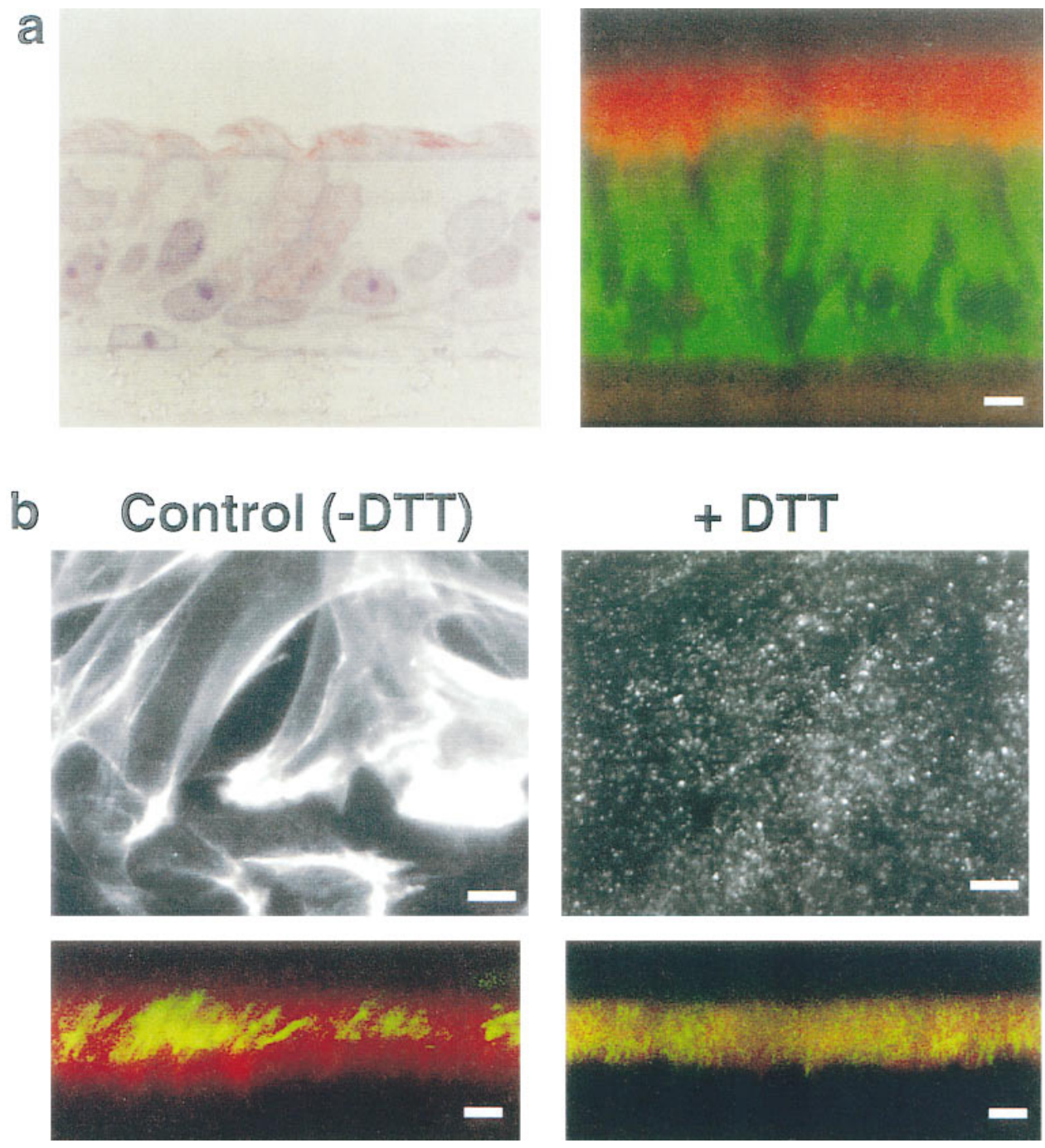
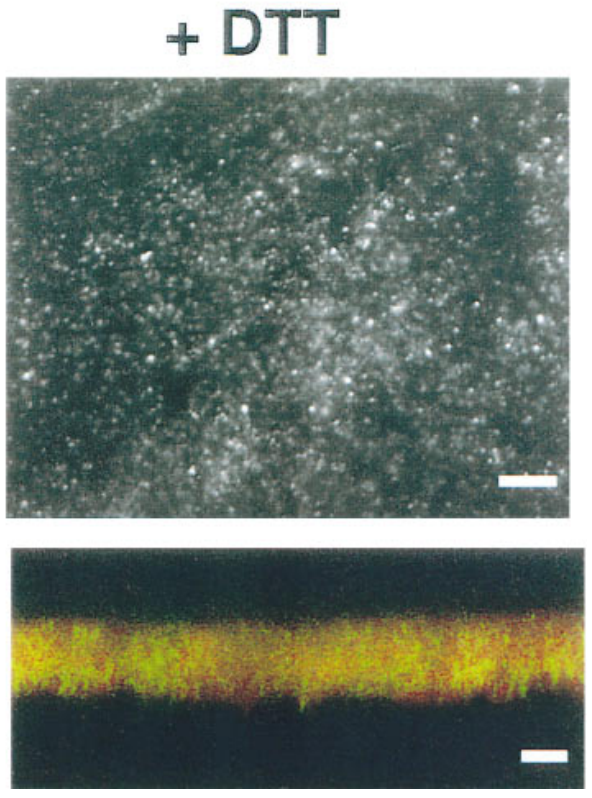

Figure 1. Identification of the mucus and periciliary fluid layers in the luminal surface liquid of cultures of hTBE cells. $(a$, left $)$ Light micrograph of a hTBE culture demonstrating well-differentiated ciliated and goblet cells. (right) Confocal micrograph $(40 \times / 1.0 \mathrm{NA}$ objective) of a living hTBE culture. The cells were stained with calcein/AM (green) and the luminal compartment with Texas Redconjugated dextran $(10,000$ daltons). Scale $=10 \mu \mathrm{m}$. (b) Images of the surface liquid layer labeled with fluorescent microspheres (0.02 vol\%) acquired before $(-D T T)$ and after $(+D T T)$ treating the cultures with dithiothreitol (10 $\mathrm{mM}, 5 \mathrm{~min}$ ) and washing with PBS. Images acquired (top) at low magnification by conventional fluorescent microscopy $(5 \times$ objective; scale $=0.2 \mathrm{~mm})$, or (bottom) at high magnification by xz scanning confocal microscopy $(40 \times / 1.0$ NA objective). The culture in the bottom panels was colabeled with Texas Red-conjugated dextran. Scale $=10 \mu \mathrm{m}$. 
Image quantification was achieved using a MetaMorph image analysis workstation (Universal Imaging, West Chester, PA). All data are expressed as the mean \pm SE.

\section{Results}

Well-differentiated cultures of hTBE cells (Fig. $1 a$, left) were used to test whether PCL is transported during mucociliary clearance. A unique capacity to study the ASL topography is afforded by the vertical (xz) sectioning capacity of the confocal microscope, which produces cross-sectional, profile images of ASL. In the xz images from the confocal microscope, the surface liquid of the cultures stained homogeneously with Texas Red-conjugated dextran $(10,000 \mathrm{D})$, and the dye front coincided with the apical membrane of the calcein-labeled cells (Fig. $1 a$, right). The depth of the surface liquid following the addition of the tracer ranged from the $6-\mu \mathrm{m}$ height of cilia to $>100 \mu \mathrm{m}$, with depths $>6 \mu \mathrm{m}$ associated with the presence of mucus overlying the PCL layer. This range of ASL depths is similar to those reported for airways surface liquid in vivo (24).

The topology and movement of the mucus layer in the cultures were visualized with fluorescent microspheres. By conventional fluorescence microscopy, microspheres $0.2 \mu \mathrm{m}$ in diameter revealed organized sheets of mucus in the ASL compartment of the cultures (Fig. $1 \mathrm{~b}$, left). In the vertical profile images (xz) from the confocal microscope, the microspherelabeled mucus layer overlaid the PCL (Fig. $1 b$, left). Association of the microspheres with mucus was indicated (Fig. $1 b$, right) by their dispersal with DTT, which breaks apart the linear mucin polymers comprising the scaffolding of the mucus gel (25). After this treatment, the microspheres were observed to distribute throughout the surface liquid layer, including the periciliary liquid.

In $\sim 25 \%$ of the cultures examined, $1-\mu \mathrm{m}$ fluorescent microspheres labeling the mucus layer exhibited a steady rotation (Fig. $2 a$ ). This rotational mucus transport persisted for periods of 1 or more weeks, and it indicated that the ciliated cells had spontaneously oriented and coordinated their ciliary activities, in vitro, during this period. Removing the mucus from these cultures with DTT and a thorough washing abolished the organized movement of microspheres. As noted in Methods, this treatment had no affect on the measured frequency of ciliary beating; $24 \mathrm{~h}$ later, the cultures again exhibited microsphere rotation due to the presence of newly secreted mucus (data not shown). The dependence of particle transport on the presence of mucus in hTBE cultures is consistent with measurements of mucociliary clearance both in vitro and in vivo (26-29).

Invariably, rotating microspheres were observed to move in formation, suggesting that the mucus in which they were entrapped moved as a network. This notion was confirmed by the observation that the microspheres were transported at a constant angular velocity (Fig. $2 b$ ). The mean maximal microsphere velocity at the outer edge of the rotating mucus network was $84.2 \pm 11.2 \mu \mathrm{m} / \mathrm{sec}(n=5)$, which is similar to the rates of mucociliary clearance measured non-invasively in human trachea $(66-83 \mu \mathrm{m} / \mathrm{s})(1)$.

Cultures exhibiting rotational mucus transport were used to study the behavior of PCL during mucociliary transport. Caged fluorescein-dextran contained in the surface liquid layer was photoactivated to generate vertical, fluorescent columns (400 $\mu \mathrm{m}$ in diameter), at points midway along the radius of mucus rotation. When studied en face at low magnification a
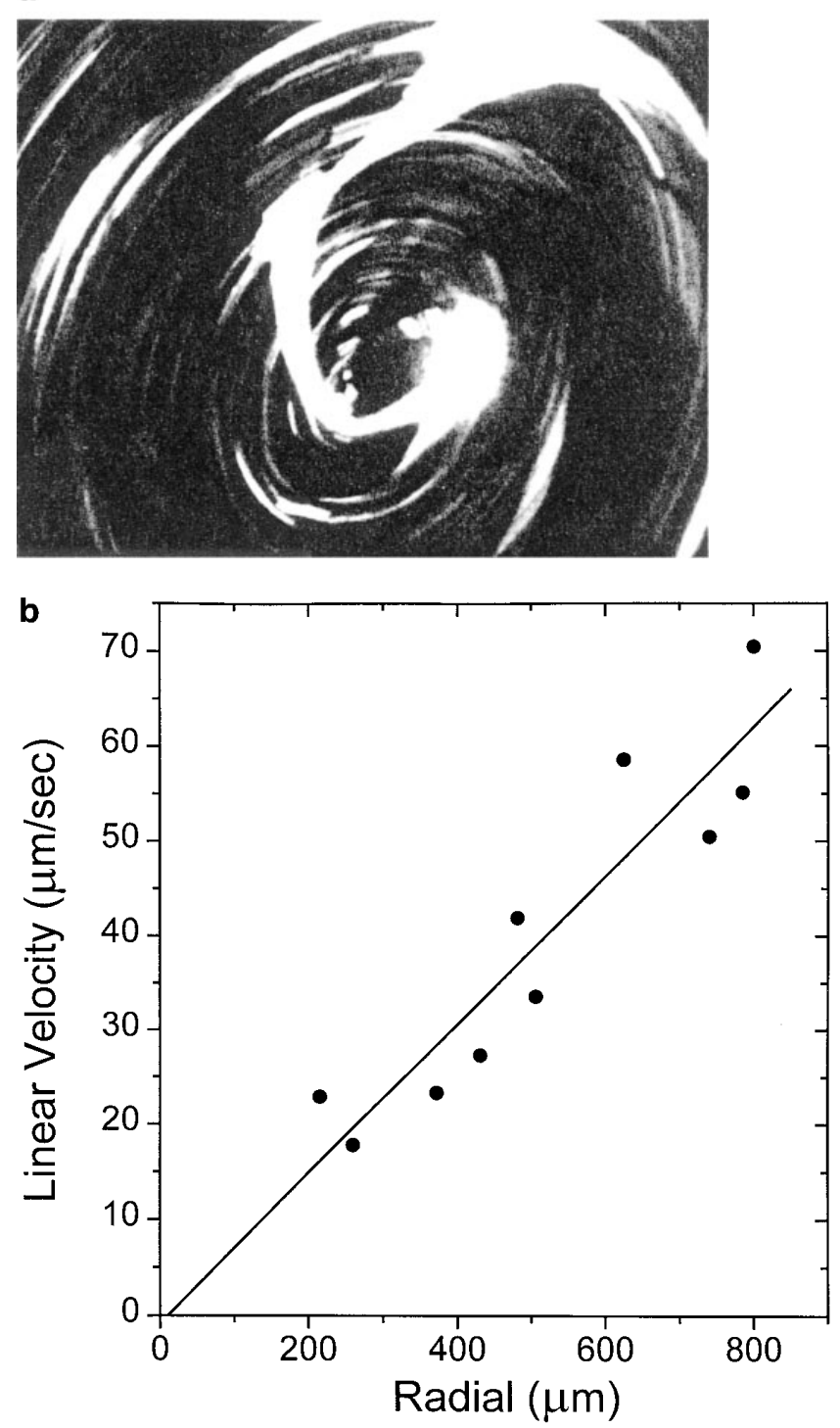

Figure 2. Mucus transport by hTBE cultures. (a) The coordinated action of cilia in hTBE cultures was identified by the directional movement of 1- $\mu \mathrm{m}$ fluorescent microspheres in the surface liquid. The image shown was acquired as a single 5-s exposure; the streaks represent the paths of individual microspheres $(5 \times$ objective; field diameter $\sim 2 \mathrm{~mm}$ ). (b) Linear velocities of fluorescent microspheres calculated from $(a)$ were plotted against the distance from the center of the rotation (radial).

( $5 \times$ objective) by conventional fluorescence microscopy (Fig. $3 a$ ), the areas of released dextran fluorescence were observed to move at a rate of $39.2 \pm 4.7 \mu \mathrm{m} / \mathrm{s}(n=13)$. The direction of this movement always matched the direction of mucus rotation and the velocity compared well with the velocity of microspheres at the same radial position $(39.8 \pm 4.2 \mu \mathrm{m} / \mathrm{s})$. Significantly, the spots of released fluorescence did not elongate or smear as they moved, suggesting that the mucus and PCL layers within the labeled region of surface liquid moved as a unit. The movement of the released fluorescent dextrans was decreased $>80 \%$, to $4.82 \pm 0.6 \mu \mathrm{m} / \mathrm{s}(n=12)$ following DTT treatment and washing with PBS. Hence, the movement of fluorescent dextran in the PCL layer depended upon the pres- 

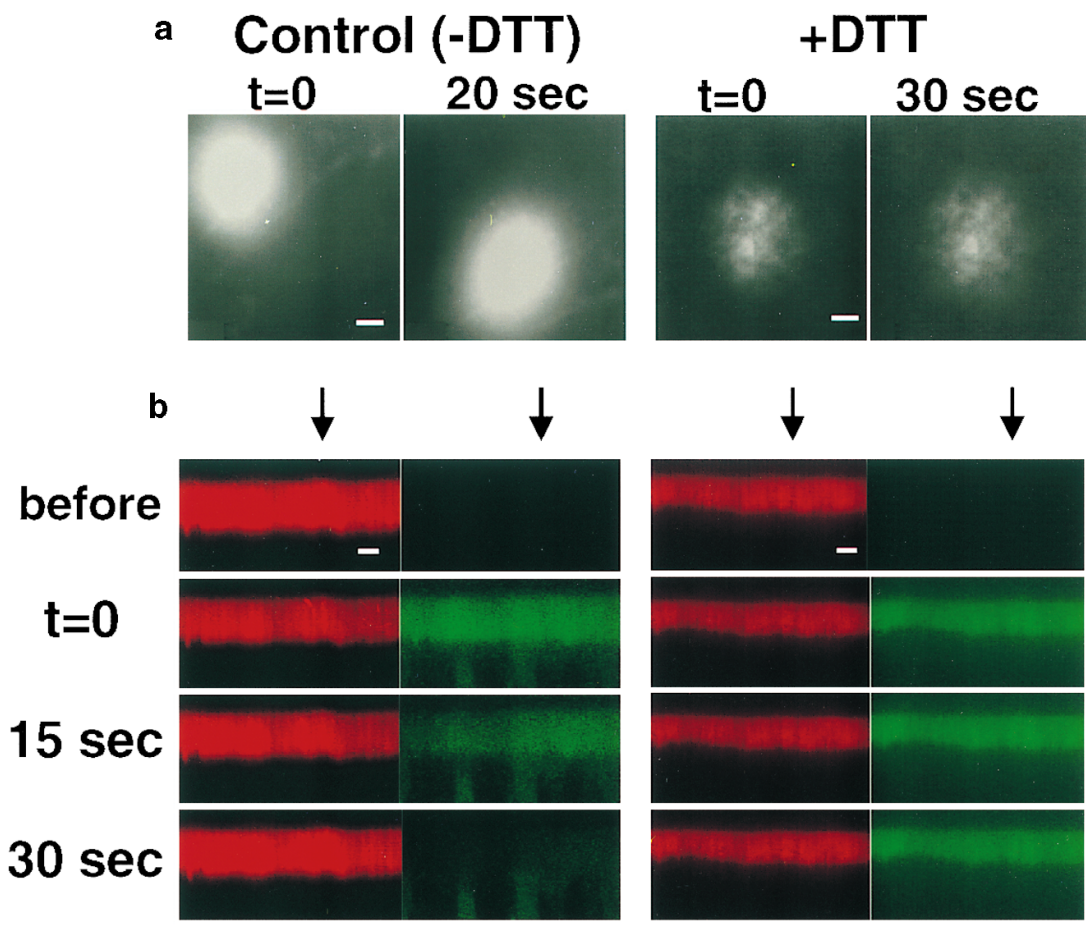

Control (-DTT)
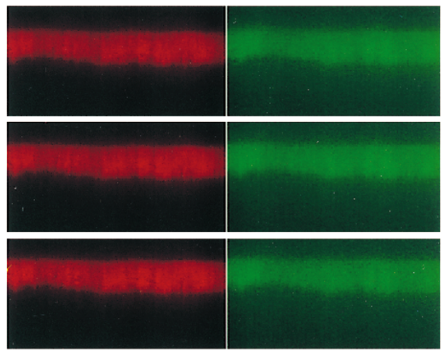

+ DTT

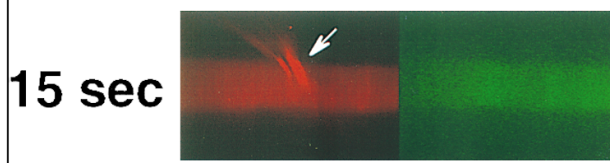

\section{Control with micro- spheres included}

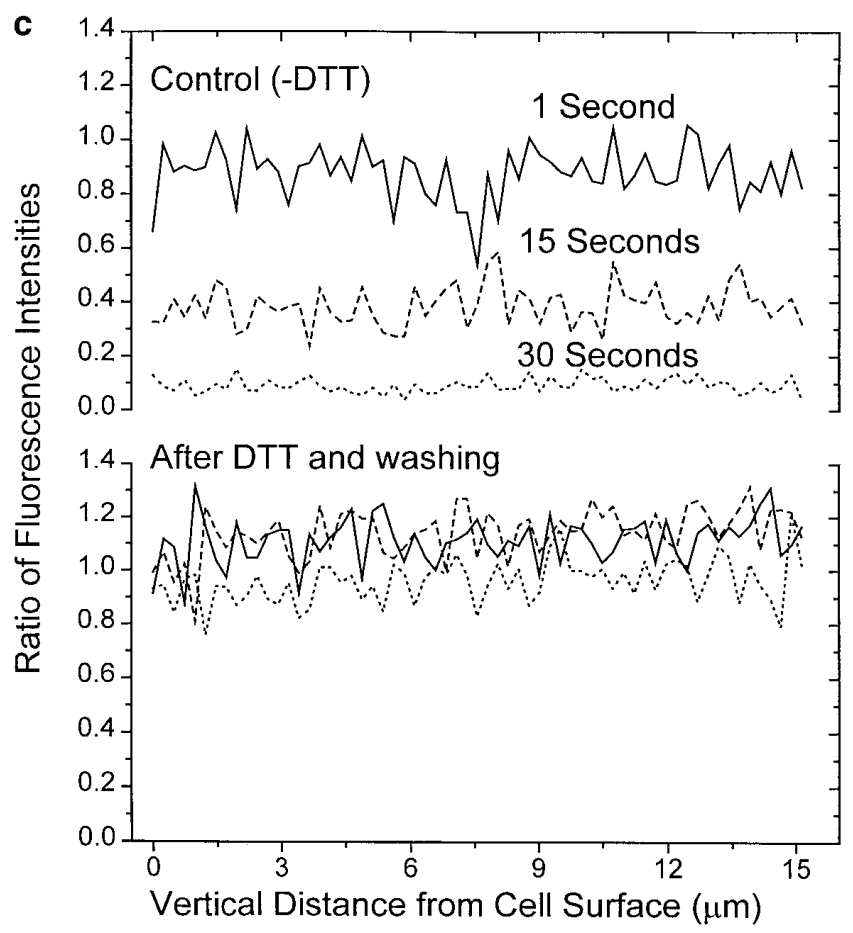

Figure 3. Transport of surface liquid by mucociliary transport revealed by photoactivation of caged fluorescein-conjugated dextrans. hTBE culture surface liquid was labeled with caged fluorescein-conjugated dextran $(10,000 \mathrm{D})$, and the fate of released dextran fluorescence was determined following photoactivation. (a) Conventional microscopy at low power $(5 \times$ objective; scales $=0.2 \mathrm{~mm})$. $($ left $)$ Control $(-D T T)$ shows migration of the released fluorescent dextran during the 20-s period of observation. (right) After removing the mucus layer $(+D T T)$, the migration of released fluorescent dextran was minimal. (b) xz scanning confocal microscopy at high power $(40 \times / 1.0 \mathrm{NA}$ objective; scales $=$ $10 \mu \mathrm{m}$ ) in cultures colabeled with Texas Red-conjugated dextran. (left) Control $(-D T T)$, showing the migration, out of frame, of released fluorescence dextran (green) in both the PCL and mucus layers of the surface liquid. (right) After removal of the mucus layer $(+D T T)$ there was no detectable change in released dextran fluorescence in the PCL. (inset) Additional 15-s control preparation in which fluorescent microspheres were included in the ASL. The beads appear as slanted streaks (arrow) as a result of their movement during image acquisition. The streaks "point" to the right, the direction of ASL movement. (c) Profiles of fluorescence intensities along the verticals indicated by arrows in $(b)$. Values presented as the ratio of released dextran fluorescence to Texas Red fluorescence (confocal microscope photomultipliers adjusted to ratio $\sim 1.0$ at the beginning of the experiment). 
ence and movement of a mucus gel. Notably, the transport rate of surface liquid observed following removal of the mucus gel approximated closely that predicted for PCL by theoretical treatments of ciliary beating alone $(13,14)$.

Confocal microscopy was used to visualize with vertical profile sectioning $(\mathrm{xz})$ the behavior of released fluorescent dextrans within the surface liquid layer. A small vertical domain of the ASL compartment at the trailing edge of the released column of fluorescence (e.g., the "spot" in Fig. $3 a$ ) was visualized with the same $40 \times$ objective in $\mathrm{xz}$ sections, and the distribution of a uniform (non-photoactivated) marker of ASL (TR-dextran) was compared with the released ("uncaged") green fluorescence. In the presence of mucus (control), the green fluorescence intensity in PCL layer decreased rapidly with time after activation due to the transport of the released fluorescein-dextran away from the scanned area (Fig. $3 b$ ). Fluorescent microspheres were generally left out of the solutions applied to the luminal surface in these experiments to avoid interference in quantification of the TR-dextran. When microspheres were included, however, their movement during the scanning process yielded a diagonal streak in the resulting image, which indicated that the mucus layer was being transported (Fig. $3 \mathrm{~b}$, inset). In 12 measurements at different radial positions in four cultures, $88.8 \pm 3.7 \%$ of the released fluorescein dextran was cleared from both the mucus and PCL layers within $30 \mathrm{~s}$, and there was no detectable difference in the clearance rates for the two layers (Fig. $3 c$ ). In contrast, after removing mucus with DTT, the clearance of released dextran fluorescence was slowed significantly to $21.1 \pm 12.7 \%$ within 30 $\mathrm{s}(n=3)$. Thus, these observations confirm those made by conventional fluorescence microscopy that the photoactivated dextran fluorescence comigrates in the PCL and mucus layers.

\section{Discussion}

Theoretical assessments of ciliary propulsion of water and mucus have commonly concluded that the PCL is largely stationary, with cilia-adherent water generally being swept forward during the power stoke and backward during the recovery stroke (11-15). Only in the outermost region of the periciliary layer, in the area lying above the path swept out on the ciliary return stroke, has a modest transport of water been predicted to occur $(13,14$; Fig. $4 a$, curve $A)$. Were the PCL in fact stationary, the only scenario explaining the observed cotransport of released dextran fluorescence in the PCL and mucus layers along the epithelial surface (Fig. 3) would be the rapid diffusion of fluorescent dextran from the PCL into the mucus layer, which is selectively transported, and from which dextran diffuses back into adjacent unlabeled PCL. Because the mucus layer is, as predicted, effectively an unstirred layer (dextran diffusivities in water and in mucus were $1.6 \times 10^{-6}$ and $3.6 \times$ $10^{-8} \mathrm{~cm}^{2} / \mathrm{sec}$, respectively), this possibility is highly unlikely. Hence, we conclude that released fluorescent dextrans in the PCL moved because they were cotransported in the PCL layer efficiently along with mucus. Moreover, the dependency of PCL transport on the transport of mucus (Fig. 3) indicates an important coupling between the ciliary-driven movement of mucus and the transport of the underlying periciliary liquid.

From classical fluid dynamics, a Newtonian fluid lying between a moving slab and an immobile plane exhibits a sheardriven flow in which the velocity profile in the steady state is linear (Fig. 4, curve B). From Fig. 4, it may be appreciated that a
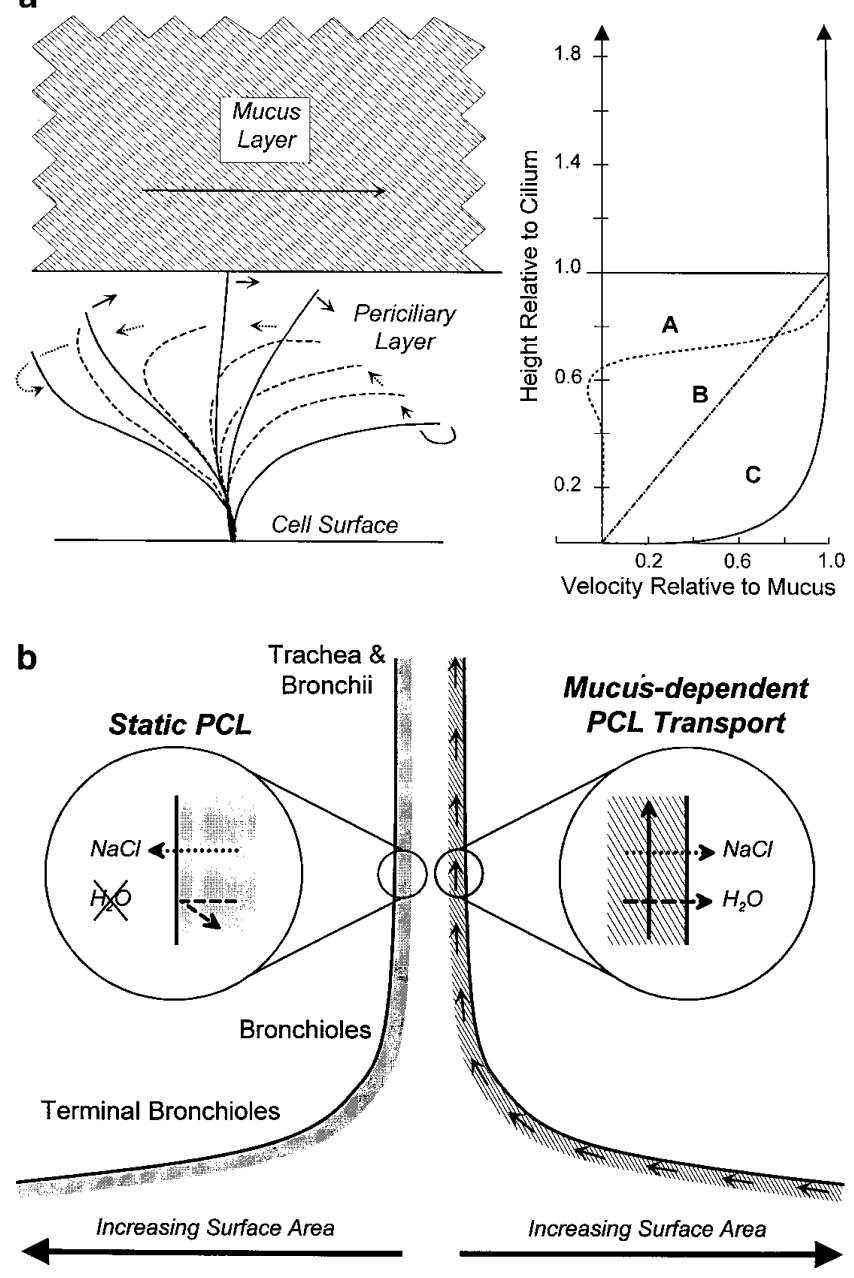

Figure 4. Models of axial surface liquid and transepithelial transport in the lung. (a) Lateral fluid velocity profiles in airway surface liquid predicted for three different considerations, with the ordinate aligned to the diagram of the ciliary beat cycle at the left. Curve $A$ approximates velocity profiles in the PCL predicted from theoretical considerations of ciliary propulsion of mucus $(13,14)$. Note nominally zero velocities at $70-75 \%$ of the ciliary length, below the level of the return stroke. Curve $B$ depicts the velocity profile predicted for PCL flow driven solely by frictional interactions with the mucus layer. Curve $C$ depicts the velocity profile predicted for the PCL from the observations in this work that the flow of the PCL and mucus layers are essentially indistinguishable. (b) Two models by which airway surface liquid depth might be maintained considering the convergent surface area over which mucus is transported cephalad during mucociliary clearance. The lung is represented as an inverted funnel with the ordinate and abscissa scaled to approximate the heights and cumulative surface areas of the different regions comprising the human lung. Right and left sides depict models favoring axially transported (10) and stationary $(8,9)$ periciliary liquid layers, respectively. Solid arrows indicate transport of PCL, and dotted and dashed arrows indicate the transepithelial transport of $\mathrm{NaCl}$ and/or water necessary to account for a constant ASL depth in the two models.

the mean velocity of PCL transport predicted for ciliary propulsion alone (Fig. 4, curve $A$ ) is $\sim 20 \%$ that of mucus, and for pure shear-related propulsion it is $50 \%$. The PCL velocity observed, however, was significantly higher than either of these rates: by conventional microscopy, the leading edge of areas of released dextran fluorescence had essentially the same velocity 
as nearby mucus-entrapped microspheres (Fig. $3 a$ ); and by confocal microscopy, the clearance rates of dextran in the PCL and mucus layers were indistinguishable (Fig. 3, $b$ and $c$ ). Because the observed coupling between the movement of the mucus layer and the underlying PCL is more efficient than can be explained by simple frictional interations (Fig. $4 a$, curve $C$ ), we suggest that mixing of the PCL by cilia promotes the transfer of momentum from the mucus layer to the PCL, analogous to the enhanced transfer of heat, matter, and momentum in well-mixed systems.

Several aspects of our techniques and analyses deserve comment. First, our system models ASL-epithelial physiology in the absence of lumenal air flow and submucosal blood flow. In the time domain of our experiments ( $30 \mathrm{~s})$, it is unlikely that absence of air/blood flow perturbs our conclusions regarding PCL transport. However, blood flow likely is particularly important in vivo for removing liquid absorbed across the epithelium to adjust ASL volume (see below) from the submucosal space to maintain hydrostatic and oncotic forces in the compartment constant. Second, our analysis of mucus PCL coupling predicts "no-slip" conditions at the boundary between the cell apical membranes and PCL. The apical membranes likely support an unstirred ("no-slip") layer, in part organized by the glycocalyx $(0.1 \mu \mathrm{m})$ in height, but this layer cannot be resolved using light microscopy (limit of resolution $=0.2 \mu \mathrm{m}$ ). Finally, we cannot exclude the possibility of mucus threads extending into the PCL and providing, at least partially, the observed coupling of transport rates between the two layers. We have attempted, without success, to visualize such threads using a variety of fixation and glycoconjugate staining techniques for both light and transmission electron microscopy (5). A definitive answer to this question, however, must await the development of suitable probes, e.g., high-affinity, mucin-specific antibodies.

The observation that PCL is transported along the surface of well-differentiated cultures of airway epithelial cells leads us to predict that the axial coordination of ciliary beating in the mammalian lung in vivo propels not only mucus but also the underlying PCL from the lung toward the mouth. There are important implications for the macroscopic and epithelial physiology of the conducting airways and related pathological processes which result from PCL transport along airway surfaces.

Viewed from the macroscopic level of coordination of ASL metabolism within all regions of the airways, given an aggregate ciliated surface area of $2,400 \mathrm{~cm}^{2}$ in the airways (30), an average ciliary length of $5 \mu \mathrm{m}(31)$, and a mucociliary transport rate of $0.5 \mathrm{~cm} / \mathrm{min}$ (32), the approximate volume of PCL potentially transported cephalad in the human lung is $864 \mathrm{ml}$ per d. As postulated originally by Kilburn (10), were epithelial absorptive activities not to occur, the PCL layer would increase in thickness during axial distal to proximal airways transport over a diminishing surface area, thereby compromising air flow. Consequently, transport of PCL is consistent with previous reports of net absorption of salt and liquid from airway surfaces as depicted in Figure $4 b(6,33,34)$. The majority of the liquid moving proximally is absorbed and removed from the lung via the bronchial circulation, with $<10-100 \mathrm{ml}$ of total ASL appearing at the mouth, as indicated by data from patients with tracheostomies (35).

Viewed from the perspective of lung defense, axial PCL transport provides an effective mechanism for clearance from the lung of inhaled hydrophilic toxicants. If PCL were stationary, inhaled insoluble particulates trapped in mucus would be cleared from the lungs, whereas inhaled hydrophilic toxicants might remain in the lung for extended periods of time. Axial PCL transport provides the mechanism to clear the lung efficiently of this class of toxic substances.

Finally, with respect to the relationship of PCL physiology to epithelial function in disease, as described above, a recently proposed hypothesis (9) holds that (a) ASL normally is hypotonic due to the absorption of salt but not liquid by the airways epithelium, and (b) CF epithelium, due to a defect in cellular $\mathrm{Cl}^{-}$conductance, cannot extract $\mathrm{NaCl}$ from ASL, thereby rendering salt-sensitive antimicrobial peptides inactive and promoting infection. Implicit aspects of this hypothesis (8) are that the epithelium is normally impermeable to water (so that surface liquid can be maintained hypotonic) and that PCL is stationary (because volume absorption cannot be a feature of a water-impermeable epithelium). Our observation that PCL is transported axially in the lungs, coupled with reported significant airway epithelial water permeabilities $(34,36)$, argues against this hypothesis. Indeed, we speculate that CF pathogenesis reflects, in part, airways plugging and recurrent infection due to excessive volume absorption and consequent abnormally low axial movement of PCL (and mucus), especially in small airways. This conclusion suggests that novel therapies for CF lung disease should not focus on perturbing ASL tonicity, but rather on restoring ASL volume.

\section{Acknowledgments}

The authors are deeply appreciative of Dr. Kazushige Yano and Dr. Toshihiro Kameda for the technical discussions that helped shape the conclusions.

This work was supported by grants from the National Institutes of Health (HL34322, HL42384) and the Cystic Fibrosis Foundation (RO26).

\section{References}

1. Salathe, M., T.G. O'Riordan, and A. Wanner. 1997. Mucociliary clearance. In The Lung: Scientific Foundations. R.G. Crystal, J.B. West, P.J. Barnes, and E.R. Weibel, editors. Lippincott-Raven, Philadelphia. 2295-2308.

2. Lucas, A.M., and L.C. Douglas. 1934. Principles underlying ciliary activity in the respiratory tract. Arch. Otolaryngol. 20:518-541.

3. Yoneda, K. 1976. Mucous blanket of rat bronchus: an ultrastructural study. Am. Rev. Respir. Dis. 114:837-842.

4. Sanderson, M.J., and M.A. Sleigh. 1981. Ciliary activity of cultured rabbit tracheal epithelium: beat pattern and metachrony. J. Cell Sci. 47:331-347.

5. Sims, D.E., and M.M. Horne. 1997. Heterogeneity of the composition and thickness of tracheal mucus in rats. Am. J. Physiol. 273:C1036-C1041.

6. Boucher, R.C. 1994. Human airway ion transport. Part one. Am. J. Respir. Crit. Care Med. 150:271-281.

7. Boucher, R.C. 1994. Human airway ion transport. Part two. Am. J. Respir. Crit. Care Med. 150:581-593.

8. Quinton, P.M. 1994. Viscosity versus composition in airway pathology. Am. J. Respir. Crit. Care Med. 149:6-7.

9. Smith, J.J., S.M. Travis, E.P. Greenberg, and M.J. Welsh. 1996. Cystic fibrosis airway epithelia fail to kill bacteria because of abnormal airway surface fluid. Cell. 85:229-236.

10. Kilburn, K.H. 1968. A hypothesis for pulmonary clearance and its implications. Am. Rev. Respir. Dis. 98:449-463.

11. Sleigh, M.A. 1977. The nature and action of respiratory tract cilia. In Respiratory Defense Mechanisms, Part I. J.D. Brain, D.F. Proctor, and L.M. Reid, editors. Dekker, New York. 247-288.

12. Satir, P., and M.A. Sleigh. 1990. The physiology of cilia and mucocilary interactions. Annu. Rev. Physiol. 52:137-155.

13. Blake, J.R., and M.A. Sleigh. 1974. Mechanics of ciliary locomotion. Biol. Rev. 49:85-125.

14. Fulford, G.R., and J.R. Blake. 1986. Muco-ciliary transport in the lung. J. Theor. Biol. 121:381-402. 
15. King, M., M. Agarwal, and J.B. Shukla. 1993. A planar model for mucociliary transport: effect of mucus viscoelasticity. Biorheology. 30:49-61.

16. Gray, T.E., K. Guzman, C.W. Davis, L.H. Abdullah, and P. Nettesheim. 1996. Mucociliary differentiation of serially passaged normal human tracheobronchial epithelial cells. Am. J. Respir. Cell Mol. Biol. 14:104-112.

17. Wu, R., J. Yankaskas, E. Cheng, M.R. Knowles, and R. Boucher. 1985. Growth and differentiation of human nasal epithelial cells in culture. Serumfree, hormone-supplemented medium and proteoglycan synthesis. Am. Rev. Respir. Dis. 132:311-320.

18. Lechner, J.F., and M.A. LaVeck. 1985. A serum-free method for culturing normal human bronchial epithelial cells at clonal density. J. Tiss. Cult. Meth. 9:43-48.

19. Kaartinen, L., P. Nettesheim, K.B. Adler, and S.H. Randell. 1993. Rat tracheal epithelial cell differentiation in vitro. In Vitro Cell. Dev. Biol. Anim. 29A:481-492.

20. Shah, P.L., S.F. Scott, R.A. Knight, C. Marriott, C. Ranasinha, and M.E. Hodson. 1996. In vivo effects of recombinant human DNase I on sputum in patients with cystic fibrosis. Thorax. 51:119-125.

21. Vasconcellos, C.A., P.G. Allen, M.E. Wohl, J.M. Drazen, P.A. Janmey, and T.P. Stossel. 1994. Reduction in viscosity of cystic fibrosis sputum in vitro by gelsolin. Science. 263:969-971.

22. Geary, C.A., C.W. Davis, A.M. Paradiso, and R.C. Boucher. 1995. Role of CNP in human airways: cGMP-mediated stimulation of ciliary beat frequency. Am. J. Physiol. 268:Pt 1:1021-1028.

23. Yguerabide, J., J.A. Schmidt, and E.E. Yguerabide. 1982. Lateral mobility in membranes as detected by fluorescence recovery after photobleaching. Biophys. J. 39:69-75.

24. Rahmoune, H., and K.L. Shephard. 1995. State of airway surface liquid on guinea pig trachea. J. Appl. Physiol. 78:2020-2024.

25. Sheehan, J.K., D.J. Thornton, M. Somerville, and I. Carlstedt. 1991. Mu- cin structure. The structure and heterogeneity of respiratory mucus glycoproteins. Am. Rev. Respir. Dis. 144:4-9.

26. Sade, J., N. Eliezer, A. Silberberg, and A.C. Nevo. 1970. The role of mucus in transport by cilia. Am. Rev. Respir. Dis. 102:48-52.

27. Spungin, B., and A. Silberberg. 1984. Stimulation of mucus secretion, ciliary activity, and transport in frog palate epithelium. Am. J. Physiol. 247:299-308.

28. Wills, P.J., M.J. Garcia Suarez, A. Rutman, R. Wilson, and P.J. Cole. 1995. The ciliary transportability of sputum is slow on the mucus-depleted bovine trachea. Am. J. Respir. Crit. Care Med. 151:1255-1258.

29. Gatto, L.A. 1993. Cholinergic and adrenergic stimulation of mucociliary transport in the rat trachea. Respir. Physiol. 92:209-217.

30. Mercer, R.R., M.L. Russell, V.L. Roggli, and J.D. Crapo. 1994. Cell number and distribution in human and rat airways. Am. J. Respir. Cell Mol. Biol. 10:613-624.

31. Serafini, S.M., and E.D. Michaelson. 1977. Length and distribution of cilia in human and canine airways. Bull. Eur. Physiopathol. Respir. 13:551-559.

32. Yeates, D.B., N. Aspin, H. Levison, M.T. Jones, and A.C. Bryan. 1975. Mucociliary tracheal transport rates in man. J. Appl. Physiol. 39:487-495.

33. Jiang, C., W.E. Finkbeiner, J.H. Widdicombe, P.B. McCray, Jr., and S.S. Miller. 1993. Altered fluid transport across airway epithelium in cystic fibrosis. Science. 262:424-427.

34. Folkesson, H.G., M.A. Matthay, H. Hasegawa, F. Kheradmand, and A.S. Verkman. 1994. Transcellular water transport in lung alveolar epithelium through mercury-sensitive water channels. Proc. Natl. Acad. Sci. USA. 91:4970 4974.

35. Toremalm, N.G. 1960. The daily amount of tracheo-bronchial secretions in man. Acta Oto-Laryngol. Suppl. 158:43-53.

36. Folkesson, H.G., M.A. Matthay, A. Frigeri, and A.S. Verkman. 1996. Transepithelial water permeability in microperfused distal airways. Evidence for channel-mediated water transport. J. Clin. Invest. 97:664-671. 